\title{
Proton structure from a light-front Hamiltonian
}

\author{
Chandan Mondal $\oplus^{1,2,6, *}$ Siqi Xu๑ ${ }^{1,2,6, \dagger}$ Jiangshan Lan, ${ }^{1,2,3,6,+}$ Xingbo Zhao $\oplus^{1,2,6, \S}$ Yang Li ${ }^{2,4, \|}$ \\ Dipankar Chakrabarti, ${ }^{5, \uparrow}$ and James P. Vary $\odot^{4, * *}$ \\ (BLFQ Collaboration) \\ ${ }^{1}$ Institute of Modern Physics, Chinese Academy of Sciences, Lanzhou 730000, China \\ ${ }^{2}$ School of Nuclear Science and Technology, University of Chinese Academy of Sciences, \\ Beijing 100049, China \\ ${ }^{3}$ Lanzhou University, Lanzhou 730000, China \\ ${ }^{4}$ Department of Physics and Astronomy, Iowa State University, Ames, Iowa 50011, USA \\ ${ }^{5}$ Department of Physics, Indian Institute of Technology Kanpur, Kanpur 208016, India \\ ${ }^{6}$ CAS Key Laboratory of High Precision Nuclear Spectroscopy, Institute of Modern Physics, Chinese \\ Academy of Sciences, Lanzhou 730000, China
}

(Received 27 November 2019; revised 5 April 2020; accepted 29 June 2020; published 14 July 2020)

\begin{abstract}
We obtain the electromagnetic form factors, the axial form factor, and the parton distribution functions of the proton from the eigenstates of a light-front effective Hamiltonian in the leading Fock representation suitable for low-momentum scale applications. The electromagnetic and the axial form factors are found to be in agreement with the available experimental data. The unpolarized, the helicity, and the transversity valence quark distributions, after QCD evolution, are consistent with the global QCD analyses. The tensor charge also agrees the experimental data, while the axial charge is somewhat outside the experimental error bar.
\end{abstract}

DOI: $10.1103 /$ PhysRevD.102.016008

\section{INTRODUCTION}

Electromagnetic form factors (FFs) and parton distribution functions (PDFs) have taught us a great deal about the internal structure of the proton. The Fourier transform of these FFs provides information about spatial distributions of the proton's constituents [1,2]. Well-known examples include the charge and magnetization distributions. Another essential tool to investigate hadron structure is deep inelastic scattering (DIS), where individual quarks are resolved. One can extract the PDFs from DIS, which encode the distribution of longitudinal momentum and polarization carried by the constituents. With an effective Hamiltonian for constituent quarks, suitable for low-resolution probes, we

\footnotetext{
*mondal@impcas.ac.cn xsq234@impcas.ac.cn \#jiangshanlan@impcas.ac.cn

\$xbzhao@impcas.ac.cn

"leeyoung@iastate.edu

Tipankar@iitk.ac.cn

jvary@iastate.edu
}

Published by the American Physical Society under the terms of the Creative Commons Attribution 4.0 International license. Further distribution of this work must maintain attribution to the author(s) and the published article's title, journal citation, and DOI. Funded by SCOAP ${ }^{3}$. solve for the proton's light-front wave functions (LFWFs) used to produce the FFs and the PDFs. We compare our FFs with experimental data. We apply QCD evolution to our initial PDFs to incorporate degrees of freedom relevant to higher-resolution probes which allows us to compare our QCD-evolved PDFs with global fits to experimental data.

The matrix element of the electromagnetic current for the nucleon requires two independent FFs: the Dirac and Pauli FFs. We refer to Refs. [3-7] for reviews of the experimental results and models on this subject. The nucleon electromagnetic FFs have been theoretically investigated in Refs. [8-27], while their flavor decomposition has been reported in Refs. [28-30]. Our work is motivated by the advent of high precision measurements of both proton and neutron FFs from ongoing and forthcoming experiments at Jefferson Lab [31-37].

PDFs reveal the internal structure of the nucleon in terms of number densities of confined quarks and gluons. At first approximation ("leading twist"), the spin structure of the nucleon is described in terms of three independent PDFs: the unpolarized $f_{1}(x)$, the helicity $g_{1}(x)$, and the transversity $h_{1}(x)$, where $x$ is the light-front longitudinal momentum fraction of the nucleon carried by quarks of flavor $q$. We provide these PDFs to assist in the analysis and interpretation of scattering experiments now and in the LHC era.

While the unpolarized and the helicity PDFs are fairly well determined [38-71], much less information is 
available on the transversity PDF. This distribution is important since it encodes the correlation between the transverse polarization of the constituents and the transverse polarization of the nucleon [72-81]. One of our aims is to predict the transversity PDF, compare with available data, and help motivate future challenging experiments.

Here, we adopt an effective light-front Hamiltonian between quarks and solve for its mass eigenstates at the scale suitable for low-resolution probes with the theoretical framework of basis light-front quantization (BLFQ) [82-85]. Our Hamiltonian includes the holographic QCD confinement potential [22] supplemented by the longitudinal confinement $[85,86]$ along with the one-gluon exchange (OGE) interaction [85] to account for the dynamical spin effects. By solving this Hamiltonian for the LFWFs in the constituent valence quark Fock space, and fitting the quark mass, confining strength, and coupling constants, we obtain good quality descriptions of its electromagnetic and axial FFs, radii, PDFs, axial and tensor charges.

\section{PROTON WAVE FUNCTIONS FROM AN EFFECTIVE HAMILTONIAN}

The structures of hadronic bound states are encoded in the LFWFs which are obtained as the eigenfunctions of the light-front (LF) eigenvalue equation: $H_{\mathrm{eff}}|\Psi\rangle=M^{2}|\Psi\rangle$, where $H_{\text {eff }}$ is the effective Hamiltonian of the system with the mass squared $\left(M^{2}\right)$ eigenvalue. In general, $|\Psi\rangle$ is the eigenvector in the Hilbert space spanned by all Fock sectors. At the initial scale where the proton is described by three active quarks, we adopt the LF effective Hamiltonian $H_{\text {eff }}$ defined by

$$
\begin{aligned}
H_{\mathrm{eff}}= & \sum_{a} \frac{\vec{p}_{\perp a}^{2}+m_{a}^{2}}{x_{a}} \\
& +\frac{1}{2} \sum_{a \neq b} \kappa^{4}\left[x_{a} x_{b}\left(\vec{r}_{\perp a}-\vec{r}_{\perp b}\right)^{2}-\frac{\partial_{x_{a}}\left(x_{a} x_{b} \partial_{x_{b}}\right)}{\left(m_{a}+m_{b}\right)^{2}}\right] \\
& +\frac{1}{2} \sum_{a \neq b} \frac{C_{F} 4 \pi \alpha_{s}}{Q_{a b}^{2}} \bar{u}_{s_{a}^{\prime}}\left(k_{a}^{\prime}\right) \gamma^{\mu} u_{s_{a}}\left(k_{a}\right) \bar{u}_{s_{b}^{\prime}}\left(k_{b}^{\prime}\right) \gamma^{\nu} u_{s_{b}}\left(k_{b}\right) g_{\mu \nu},
\end{aligned}
$$

where $\sum_{a} x_{a}=1$, and $\sum_{a} \vec{p}_{\perp a}=0 . m_{a / b}$ is the mass of the quark, and $\kappa$ is the strength of the confinement. $x_{a}$ represents the LF momentum fraction carried by quark $a$. Meanwhile, $\vec{p}_{\perp}$ is the relative transverse momentum, while $\vec{r}_{\perp}=\vec{r}_{\perp a}-\vec{r}_{\perp b}$, related to the holographic variable [22], is the transverse separation between two quarks. The last term in the effective Hamiltonian represents the OGE interaction where $Q_{a b}^{2}=-q^{2}=-(1 / 2)\left(k_{a}^{\prime}-k_{a}\right)^{2}-(1 / 2)\left(k_{b}^{\prime}-k_{b}\right)^{2}$ is the average momentum transfer squared. $C_{F}=-2 / 3$ is the color factor, $g_{\mu \nu}$ is the metric tensor and $\alpha_{s}$ is the coupling constant. Here, $u_{s_{a}}\left(k_{a}\right)$ is the solution of the Dirac equation, with the subscript $s_{a}$ representing spin and $k_{a}$ is the momentum of quark $a$.
Following BLFQ, we expand $|\Psi\rangle$ in terms of the two dimensional harmonic oscillator ("2D-HO") basis in the transverse direction and the discretized plane-wave basis in the longitudinal direction $[82,83]$. Each single-quark basis state is identified using four quantum numbers, $\bar{\alpha}=\{k, n, m, \lambda\}$. The longitudinal momentum of the particle is characterized by the quantum number $k$. The longitudinal coordinate $x^{-}$is confined to a box of length $2 L$ with antiperiodic boundary conditions for fermions. As a result, the longitudinal momentum $p^{+}=2 \pi k / L$ is discretized, where the dimensionless quantity $k=\frac{1}{2}, \frac{3}{2}, \frac{5}{2}, \ldots$. All many-body basis states are selected to have the same total longitudinal momentum $P^{+}=\sum_{i} p_{i}^{+}$, where the sum is over the quarks. We rescale $P^{+}$using $K=\sum_{i} k_{i}$ such that $P^{+}=\frac{2 \pi}{L} K$. For a given quark $i$, the longitudinal momentum fraction $x$ is defined as $x_{i}=p_{i}^{+} / P^{+}=k_{i} / K$.

The quantum numbers, $n$ and $m$, denote radial excitation and angular momentum projection, respectively, of the particle within the $2 \mathrm{D}-\mathrm{HO}$ basis, $\phi_{n m}\left(\vec{p}_{\perp}\right)[82,83]$. The 2D-HO basis should form an efficient basis for systems subject to QCD confinement. For the quark spin, $\lambda$ is used to label the helicity. Our multibody basis states have fixed values of the total angular momentum projection $M_{J}=\sum_{i}\left(m_{i}+\lambda_{i}\right)$.

The valence wave function in momentum space is then expanded as:

$$
\begin{aligned}
\Psi_{\left\{x_{i}, \vec{p}_{\perp i}, \lambda_{i}\right\}}^{M_{\lambda_{i}}}= & \sum_{\left\{n_{i} m_{i}\right\}}\left\{\psi\left(\left\{\bar{\alpha}_{i}\right\}\right) \prod_{i=1}^{3} \frac{1}{b}\left(\frac{\left|\vec{p}_{\perp i}\right|}{b}\right)^{\left|m_{i}\right|}\right. \\
& \times \sqrt{\frac{4 \pi \times n_{i} !}{\left(n_{i}+\left|m_{i}\right|\right) !}} e^{i m_{i} \theta_{i}} L_{n_{i}}^{\left|m_{i}\right|}\left(-\frac{\vec{p}_{\perp i}^{2}}{b^{2}}\right) \\
& \left.\times \exp \left(-\frac{\vec{p}_{\perp i}^{2}}{2 b^{2}}\right)\right\},
\end{aligned}
$$

where $\psi\left(\left\{\bar{\alpha}_{i}\right\}\right)$ is the LFWF in the BLFQ basis obtained by diagonalizing Eq. (1) numerically. $b=0.6 \mathrm{GeV}$ is the $\mathrm{HO}$ scale parameter and $\tan (\theta)=p_{2} / p_{1}$. Here $L_{n}^{|m|}$ is the associated Laguerre function. We truncate the infinite basis by introducing limit $K_{\max }$ such that, $\sum_{i} k_{i}=K_{\max }$. In the transverse direction, we also truncate by limiting $N_{\alpha}=$ $\sum_{i}\left(2 n_{i}+\left|m_{i}\right|+1\right)$ for multiparticle basis state to $N_{\alpha} \leq N_{\max }$. The basis truncation corresponds to having a UV regulator $\Lambda_{\mathrm{UV}} \sim b \sqrt{N_{\max }}$. Since we are modeling the proton at a low-resolution scale, we select $N_{\max }=10$ and $K_{\max }=16.5$. To attempt to simulate the effect of higher Fock spaces and the other QCD interactions, we use a different quark mass in the kinetic energy, $m_{\mathrm{q} / \mathrm{KE}}$ and the OGE interaction, $m_{\mathrm{q} / \mathrm{OGE}}$. Note that our approach features an effective one-gluon exchange interaction that describes short distance physics and approximately accounts for the processes where constituent quarks emit and absorb a gluon during which the system fluctuates between the $|q q q\rangle$, $|q q q g\rangle$, and higher Fock sectors. According to the mass 
evolution in renormalization group theory, the dynamical one-gluon exchange would also generate contributions to the quark mass arising from higher momentum scale leading to a decrease in the quark mass from the gluon dynamics. This leads to the suggestion that the mass in the one-gluon exchange interaction would be lighter than the kinetic mass which we associate with the long-range physics in our effective Hamiltonian. This treatment is also noted and adopted in the literature [87-89].

We set our parameters $\left\{m_{\mathrm{q} / \mathrm{KE}}, m_{\mathrm{q} / \mathrm{OGE}}, \kappa, \alpha_{s}\right\}=\{0.3 \mathrm{GeV}$, $0.2 \mathrm{GeV}, 0.34 \mathrm{GeV}, 1.1\}$ to fit the proton mass and the flavor Dirac FFs [30]. The value of $\chi^{2}$ per degree of freedom (d.o.f.) for the fit of the down quark Dirac FF is 5.9, whereas for the up quark Dirac FF the value of the $\chi^{2} /$ d.o.f. is 10.1 . For numerical convenience, we use a small gluon mass regulator $\left(\mu_{g}=0.05 \mathrm{GeV}\right)$ in the OGE interaction. We find that our results are insensitive to $\mu_{g}>0.01 \mathrm{GeV}$.

\section{FORM FACTORS AND PDFs OF THE PROTON}

In the LF formalism, the flavor Dirac $F_{1}^{q}\left(Q^{2}\right)$ and Pauli $F_{2}^{q}\left(Q^{2}\right)$ FFs in the proton can be expressed in terms of overlap integrals as [90]

$$
\begin{aligned}
& F_{1}^{q}\left(Q^{2}\right)=\int_{D} \Psi_{\left\{x_{i}^{\prime}, \vec{p}_{\perp i}^{\prime}, \lambda_{i}\right\}}^{\uparrow *} \Psi_{\left\{x_{i}, \vec{p}_{\perp i}, \lambda_{i}\right\}}^{\uparrow}, \\
& F_{2}^{q}\left(Q^{2}\right)=-\frac{2 M}{\left(q^{1}-i q^{2}\right)} \int_{D} \Psi_{\left\{x_{i}^{\prime}, \vec{p}_{\perp i}^{\prime}, \lambda_{i}\right\}}^{\uparrow *} \Psi_{\left\{x_{i}, \vec{p}_{\perp i}, \lambda_{i}\right\}}^{\downarrow},
\end{aligned}
$$

with $\int_{D} \equiv \sum_{\lambda_{i}} \int \prod_{i}\left[\frac{d x d^{2} \vec{p}_{\perp}}{16 \pi^{3}}\right]_{i} 16 \pi^{3} \delta\left(1-\sum x_{j}\right) \delta^{2}\left(\sum \vec{p}_{\perp j}\right)$. For the struck quark of flavor $q, x_{1}^{\prime}=x_{1} ; \vec{p}_{\perp 1}^{\prime}=\vec{p}_{\perp 1}+$ $\left(1-x_{1}\right) \vec{q}_{\perp}$ and $x_{i}^{\prime}=x_{i} ; \vec{p}_{\perp i}^{\prime}=\vec{p}_{\perp i}-x_{i} \vec{q}_{\perp}$ for the spectators $(i=2,3)$. We consider the frame where the momentum transfer $q=\left(0,0, \vec{q}_{\perp}\right)$, thus $Q^{2}=-q^{2}=\vec{q}_{\perp}^{2}$.

Under charge and isospin symmetry, the proton FFs can be obtained from the flavor FFs [28]: $F_{i}^{p}=e_{u} F_{i}^{u}+$ $e_{d} F_{i}^{d}$, where $e_{u}\left(e_{d}\right)=\frac{2}{3}\left(-\frac{1}{3}\right)$, with the normalizations $F_{1}^{u}(0)=2, F_{2}^{u}(0)=\kappa_{u}$ and $F_{1}^{d}(0)=1, F_{2}^{d}(0)=\kappa_{d}$ where $\kappa_{u(d)}$ is the anomalous magnetic moment of the up(down) quark. In our approach, we obtain $\kappa_{u}=1.481 \pm 0.029$ and $\kappa_{d}=-1.367 \pm 0.025$, while the extracted values from the experimental data of the proton and the neutron anomalous magnetic moments are: $\kappa_{u}^{\exp }=2 \kappa_{p}+\kappa_{n}=1.673$ and $\kappa_{d}^{\exp }=\kappa_{p}+2 \kappa_{n}=-2.033$. The nucleon Sachs FFs are again written in terms of Dirac and Pauli FFs as

$$
\begin{aligned}
& G_{E}^{p}\left(Q^{2}\right)=F_{1}^{p}\left(Q^{2}\right)-\frac{Q^{2}}{4 M_{p}^{2}} F_{2}^{p}\left(Q^{2}\right), \\
& G_{M}^{p}\left(Q^{2}\right)=F_{1}^{p}\left(Q^{2}\right)+F_{2}^{p}\left(Q^{2}\right),
\end{aligned}
$$

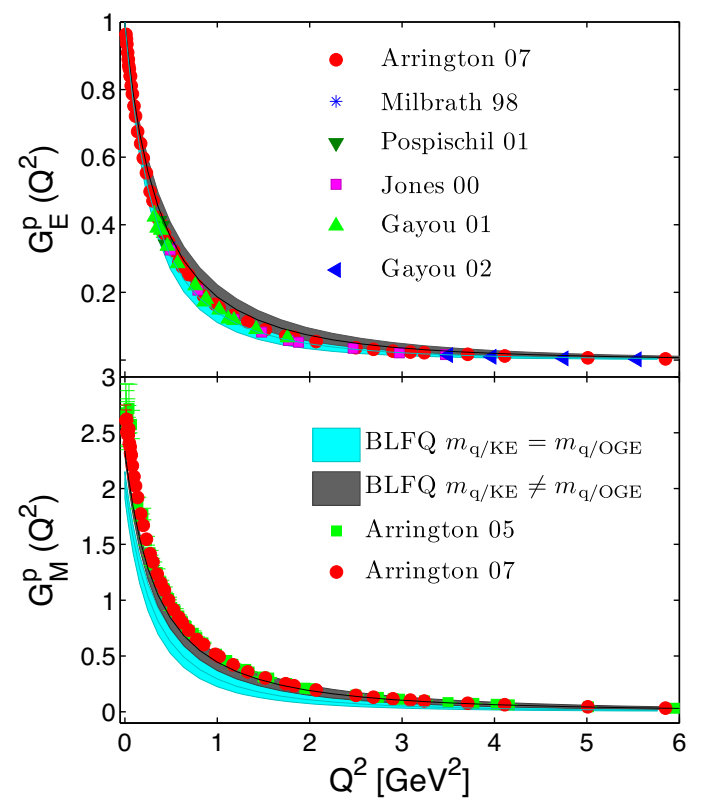

FIG. 1. Proton Sach's FFs $G_{E}^{p}\left(Q^{2}\right)$ (upper panel), and $G_{M}^{p}\left(Q^{2}\right)$ (lower panel) as functions of $Q^{2}$. The gray and cyan bands are BLFQ results, when $\left\{m_{\mathrm{KE}}, m_{\mathrm{OGE}}\right\}=\{0.3,0.2\} \mathrm{GeV}$ and $\left\{m_{\mathrm{KE}}, m_{\mathrm{OGE}}\right\}=\{0.3,0.3\} \mathrm{GeV}$ respectively, reflecting our $\alpha_{s}$ uncertainty of $10 \%$. The experimental data are taken from Refs. [91-96] and [93,97].

and the electromagnetic radii are defined by $\left\langle r_{E}^{2}\right\rangle=$ $-\left.6 \frac{d G_{E}\left(Q^{2}\right)}{d Q^{2}}\right|_{Q^{2}=0}$ and $\left\langle r_{M}^{2}\right\rangle=-\left.\frac{6}{G_{M}(0)} \frac{d G_{M}\left(Q^{2}\right)}{d Q^{2}}\right|_{Q^{2}=0}$.

In Fig. 1, we show the $Q^{2}$ dependence of the proton electric and the magnetic Sach's FFs. Overall, we obtain a reasonable agreement between theory and experiment for the proton electric FFs. At large $Q^{2}$, the magnetic form factor is also in good agreement with the data. However, our magnetic form factor at low $Q^{2}$ exhibits a small deviation from the data. We observe that $m_{\mathrm{KE}} \neq m_{\mathrm{OGE}}$ describes the form factors better than $m_{\mathrm{KE}}=m_{\mathrm{OGE}}$. It should be noted that the neglected higher Fock components $|q q q q \bar{q}\rangle$ can have a significant effect on the magnetic form factor [17].

We present our computed radii in Table I and compare with measured data $[98,99]$ as well as with recent lattice QCD calculations [27]. Here again, we find reasonable agreement with experiment.

The axial form factor, which is identified with the matrix elements of axial-vector local operator, can also be expressed in terms of LFWFs

$$
G_{A}^{q}\left(Q^{2}\right)=\int_{D}(\Lambda) \Psi_{\left\{x_{i}^{\prime}, \vec{p}_{\perp i}^{\prime}, \lambda_{i}\right\}}^{* *} \Psi_{\left\{x_{i}, \vec{p}_{\perp i}, \lambda_{i}\right\}}^{\uparrow}
$$

Here, $\Lambda=1(-1)$ depends on the struck quark helicity $\lambda_{1}=\frac{1}{2}\left(-\frac{1}{2}\right)$. Experimental information about the axial FFs is very limited. Until now, there are only two sets of 
TABLE I. Proton radii, axial and tensor charges, first moments of transversity distributions. Our results are compared with the extracted data and recent lattice QCD calculations.

\begin{tabular}{lccc}
\hline \hline Quantity & BLFQ & Extracted data & Lattice \\
\hline$r_{E} \mathrm{fm}$ & $0.802_{-0.040}^{+0.042}$ & $0.833 \pm 0.010[98]$ & $0.742(13)[27]$ \\
$r_{M} \mathrm{fm}$ & $0.834_{-0.029}^{+0.029}$ & $0.851 \pm 0.026[99]$ & $0.710(26)[27]$ \\
$r_{A} \mathrm{fm}$ & $0.680_{-0.073}^{+0.070}$ & $0.667 \pm 0.12[100]$ & $0.512(34)[101]$ \\
$g_{A}$ & $1.41_{-0.06}^{+0.06}$ & $1.2723 \pm 0.0023[99]$ & $1.271(13)[102]$ \\
$g_{T}^{d}$ & $-0.20_{-0.04}^{+0.02}$ & $-0.25_{-0.10}^{+0.30}[74]$ & $-0.204(11)[103]$ \\
$g_{T}^{u}$ & $0.94_{-0.15}^{+0.06}$ & $0.39_{-0.12}^{+0.18}[74]$ & $0.784(28)[103]$ \\
$\langle x\rangle_{T}^{u-d}$ & $0.229_{-0.048}^{+0.019}$ & $\ldots$ & $0.203(24)[104]$ \\
\hline \hline
\end{tabular}

experiments: (anti)neutrino scattering off protons or nuclei and charged pion electroproduction [105,106]. Figure 2 shows the results obtained for the axial form factor, $G_{A}=$ $G_{A}^{u}-G_{A}^{d}$ as a function of $Q^{2}$, where we compare our BLFQ results with the experimental data [106,107] and with lattice results [108]. Considering the theoretical and experimental uncertainties, the agreement is good. At $Q^{2}=0$, the axial form factor is the axial charge, $g_{A}=G_{A}(0) . g_{A}$ is quoted in Table I, where we see our value differs somewhat from extracted data [99] and lattice [102]. We also evaluate the axial radius from: $\left\langle r_{A}^{2}\right\rangle=\left.\frac{6}{g_{A}} \frac{d G_{A}\left(Q^{2}\right)}{d Q^{2}}\right|_{Q^{2}=0}$. As can be seen from the Table I, the BLFQ result is in good agreement with the extracted data from the analysis of neutrinonucleon scattering experiment $[100,109]$.

With our LFWFs, the proton's valence quark PDFs at leading twist are given by

$f_{1}^{q}=\int_{D} \Psi_{\left\{x_{i}^{\prime}, \vec{p}_{\perp i}^{\prime}, \lambda_{i}\right\}}^{\Psi_{\left\{x_{i}, \vec{p}_{\perp i}, \lambda_{i}\right\}}^{\uparrow}} \delta\left(x-x_{1}\right)$

$g_{1}^{q}=\int_{D}(\Lambda) \Psi_{\left\{x_{i}^{\prime}, \vec{p}_{\perp i}^{\prime}, \lambda_{i}\right\}}^{\uparrow *} \Psi_{\left\{x_{i}, \vec{p}_{\perp i}, \lambda_{i}\right\}}^{\uparrow} \delta\left(x-x_{1}\right)$,

$h_{1}^{q}=\int_{D}\left[\Psi_{\left\{x_{i}^{\prime}, \vec{p}_{\perp i}^{\prime}, \lambda_{i}^{\prime}\right\}}^{*} \Psi_{\left\{x_{i}, \vec{p}_{\perp i}, \lambda_{i}\right\}}^{\downarrow}+(\uparrow \leftrightarrow \downarrow)\right] \delta\left(x-x_{1}\right)$,

where the $\lambda_{1}^{\prime}=-\lambda_{1}$ and $\lambda_{2,3}^{\prime}=\lambda_{2,3}$. At the model scale relevant to constituent quark masses which are several hundred $\mathrm{MeV}$, the unpolarized PDFs for the valence quarks are normalized as $\int_{0}^{1} f_{1}^{u}(x) d x=2, \int_{0}^{1} f_{1}^{d}(x) d x=1$. We also have the following momentum sum rule: $\int_{0}^{1} x f_{1}^{u}(x) d x+\int_{0}^{1} x f_{1}^{d}(x) d x=1$. We observe that at the model scale $f_{1}^{u} / f_{1}^{d} \rightarrow 3$ and $g_{1}^{u} / g_{1}^{d} \rightarrow-7$, while the ratio for transversity PDFs, $h_{1}^{u} / h_{1}^{d} \rightarrow-4$ at $x \rightarrow 1$ region.

Next, to evolve our PDFs from our model scale, defined as $\mu_{0}^{2}$, to a higher scale $\mu^{2}$, we adopt the Dokshitzer-GribovLipatov-Altarelli-Parisi (DGLAP) equations [110-112] of QCD. This scale evolution allows quarks to emit and absorb gluons, with the emitted gluons capable of

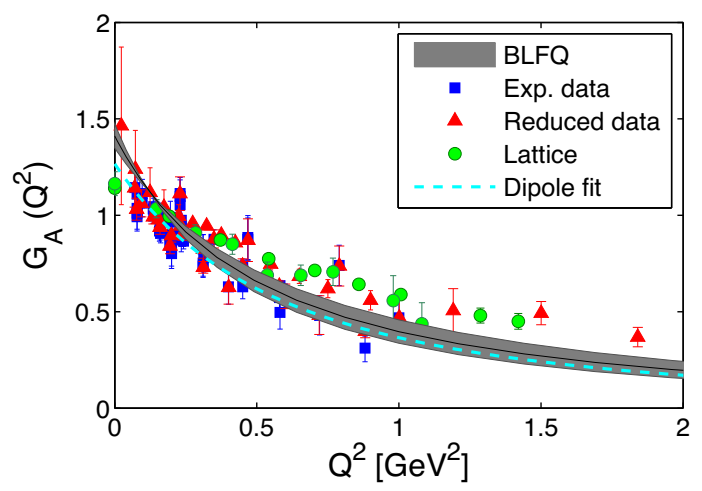

FIG. 2. Axial-vector form factor $G_{A}=G_{A}^{u}-G_{A}^{d}$ as function of $Q^{2}$. The gray band is the BLFQ result. The extracted data are taken from the Refs. [106,107] and the lattice data from [108]. The dashed line represents the dipole fit of the experimental data [106].

producing quark-antiquark pairs as well as additional gluons. In this picture, the sea quark and gluon components of the constituent quarks are revealed at the higher scale through QCD. While applying the DGLAP equations numerically [113], we impose the condition that the running coupling $\alpha_{s}\left(\mu^{2}\right)$ saturates in the infrared at a cutoff value of $\max \alpha_{s}=1$ [114,115], consistent with our fit value discussed above. Allowing $\mu_{0}^{2}$ to be different for different order of pertubative evolution, we determine them by requiring the result after evolutions to produce the total first moments of the valence quark unpolarized PDFs from the NNPDF3.0 global fit [38]. We find that $\mu_{0}^{2}$ increases when we progress to higher orders and the difference of $\mu_{0}^{2}$ between the leading order (LO), next-to-leading order (NLO), and next-to-next-to-leading order (NNLO) decreases. Also going to higher orders, the evolved PDF fits better to the NNPDF3.0 data for the second moment demonstrated in Table II.

Figure 3 shows our results for the valence quark unpolarized and spin dependent PDFs of the proton, where

TABLE II. Lowest two moments of the valence quark distributions at different scales using the LO, NLO, and NNLO perturbative evolution. BLFQ results are compared with NNPDF3.0 [38].

\begin{tabular}{lcccc}
\hline \hline Orders $\left(\mu_{0}^{2}\right)$ & Moments & $1 \mathrm{GeV}^{2}$ & $4 \mathrm{GeV}^{2}$ & $10 \mathrm{GeV}^{2}$ \\
\hline LO & $\left\langle x_{v}\right\rangle$ & 0.4673 & 0.3939 & 0.3607 \\
$\left(0.055 \mathrm{GeV}^{2}\right)$ & $\left\langle x_{v}^{2}\right\rangle$ & 0.1314 & 0.1006 & 0.0877 \\
$\mathrm{NLO}$ & $\left\langle x_{v}\right\rangle$ & 0.4751 & 0.3948 & 0.3612 \\
$\left(0.146 \mathrm{GeV}^{2}\right)$ & $\left\langle x_{v}^{2}\right\rangle$ & 0.1402 & 0.1057 & 0.0920 \\
NNLO & $\left\langle x_{v}\right\rangle$ & 0.4721 & 0.3933 & 0.3607 \\
$\left(0.195 \mathrm{GeV}^{2}\right)$ & $\left\langle x_{v}^{2}\right\rangle$ & 0.1410 & 0.1068 & 0.0935 \\
NNPDF3.0 & $\left\langle x_{v}\right\rangle$ & 0.4741 & 0.3932 & 0.3607 \\
& $\left\langle x_{v}^{2}\right\rangle$ & 0.1451 & 0.1091 & 0.0955 \\
\hline \hline
\end{tabular}


we compare the valence quark distribution after QCD evolution at NNLO with the global fits by MMHT14 [39], NNPDF3.0 [38] and CTEQ15 [40] Collaborations. The error bands in our evolved distributions are due to the spread in the initial scale $\mu_{0}^{2}=0.195 \pm 0.020 \mathrm{GeV}^{2}$ and the uncertainties in the coupling constant, $\alpha_{s}=1.1 \pm 0.1$. Our unpolarized valence PDFs for both up and down quarks are found to be in good agreement with the global fits. Meanwhile, we evolve the spin dependent PDFs from our model scale to the relevant experimental scale $\mu^{2}=$ $3 \mathrm{GeV}^{2}$ and find that the down quark helicity PDF agrees well with measured data from COMPASS Collaboration [52]. However, for the up quark, our helicity PDF tends to overestimate the data below $x \sim 0.3$.

The transversity distribution at $\mu^{2}=2.4 \mathrm{GeV}^{2}$ is also shown in Fig. 3. We compare our prediction with the global

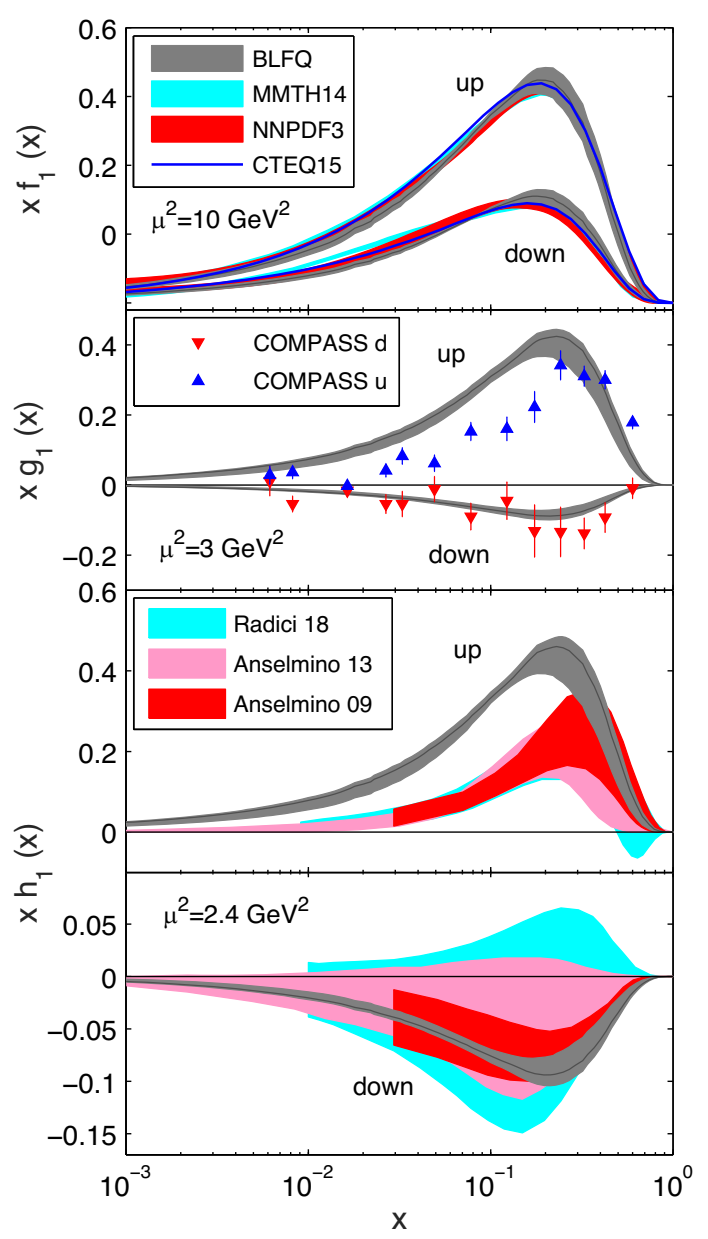

FIG. 3. Top panel: comparison for $x f_{1}(x)$ in the proton from BLFQ (gray bands) and global fits: MMHT14 [39] (cyan bands), NNPDF3.0 [38] (red bands), and CTEQ15 [40] (blue line). Second panel: comparison for $x g_{1}(x)$ in the proton from BLFQ (black bands) and measured data from COMPASS [52]. Lower two panel: Comparison for $x h_{1}(x)$ in the proton from BLFQ (gray bands) and global analyses by Radici et al. [81] (cyan bands) and Anselmino et al. [74] (pink bands), and [116] (red bands). analysis by Radici et al. [81], the global analysis by Anselmino et al. [74,116]. Our down quark transversity distribution is in accord with the fits. The up quark distribution in our approach deviates in the low $x$ region, while it shows resonable agreement at large $x$ with the global fits.

Transversity has recently received increasing attention because of the importance of a precise determination of its integral, the so-called tensor charge $g_{T}$. We compare our results at $\mu^{2}=2.4 \mathrm{GeV}^{2}$ with extracted data as well as with lattice data in Table I. Again we observe that BLFQ predicts the tensor charges quite well for down quark in comparison with the global QCD analysis [74]. However, for the up quark it deviates from the extracted data but our value is closer to recent lattice data [103] and our approach yields comparable agreement with results from phenomenological models as well as other lattice calculations [117-120]. We also provide the first moments of transversity distribution, $\langle x\rangle_{T}^{u-d}$ in the Table I, which agree reasonably well with lattice data [104].

\section{CONCLUSIONS}

We present a model for the proton that provides observables from the low resolution constituent quark scale to high resolution experiments. Specifically, we begin with an effective LF Hamiltonian incorporating confinement and one gluon exchange interaction for the valence quarks suitable for low-resolution properties. Using basis LF quantization, the LFWFs obtained as the eigenvectors of this Hamiltonian were then used to generate the proton electromagnetic and axial form factors and the initial PDFs for different quark polarizations. We have obtained reasonable agreement with the experimental data for the Sach's FFs, the axial form factor as well as the electromagnetic radii for the proton. The unpolarized, helicity and transversity PDFs at higher scale relevant to global QCD analyses have been computed based on the NNLO DGLAP equations. The initial low-resolution scale is the only adjustable parameter involved in QCD scale evolution and we obtain it by fitting the first moments of unpolarized PDFs from global QCD analyses. We then find the unpolarized, the helicity, and transversity PDFs agree with results from the corresponding global fits or experimental data in Refs. [38-40], Ref. [52], and Refs. [81,116], respectively. The axial charge and the tensor charge also show reasonable agreement with the extracted data or the lattice results. It should also be noted that basis truncation may play a role that should be examined in future research. The effective LFWFs can be used to study other parton distributions, such as the generalized parton distributions, the transverse momentum dependent parton distributions and the Wigner distributions. The presented results affirm the utility of our model and motivate application of analogous effective Hamiltonians to the other hadrons. 


\section{ACKNOWLEDGMENTS}

We thank Henry Lamm, Wei Zhu, and Shuai Liu for many useful discussions. C. M. is supported by the National Natural Science Foundation of China (NSFC) under the Grants No. 11850410436 and No. 11950410753 . X.Z. is supported by new faculty startup funding by the Institute of Modern Physics, Chinese Academy of Sciences and by Key Research Program of Frontier Sciences, CAS, Grant No. ZDBSLY-7020. This work of C. M, S.X., J. L, and X.Z. is also supported by the Strategic Priority Research
Program of the Chinese Academy of Sciences, Grant No. XDB34000000. The work of D. C. is supported by Science and Engineering Research Board under Grant No. CRG/2019/000895. J.P. V. is supported by the Department of Energy under Grants No. DE-FG0287ER40371, and No. DE-SC0018223 (SciDAC4/ NUCLEI). A portion of the computational resources were provided by the National Energy Research Scientific Computing Center (NERSC), which is supported by the Office of Science of the U.S. Department of Energy under Contract No. DE- AC02-05CH11231.
[1] G. A. Miller, Phys. Rev. Lett. 99, 112001 (2007).

[2] C. E. Carlson and M. Vanderhaeghen, Phys. Rev. Lett. 100, 032004 (2008).

[3] H. Y. Gao, Int. J. Mod. Phys. E 12, 1 (2003).

[4] C. E. Hyde-Wright and K. de Jager, Annu. Rev. Nucl. Part. Sci. 54, 217 (2004).

[5] C. F. Perdrisat, V. Punjabi, and M. Vanderhaeghen, Prog. Part. Nucl. Phys. 59, 694 (2007).

[6] S. Pacetti, R. Baldini Ferroli, and E. Tomasi-Gustafsson, Phys. Rep. 550-551, 1 (2015).

[7] V. Punjabi, C. F. Perdrisat, M. K. Jones, E. J. Brash, and C. E. Carlson, Eur. Phys. J. A 51, 79 (2015).

[8] C. Alexandrou, M. Constantinou, K. Hadjiyiannakou, K. Jansen, C. Kallidonis, G. Koutsou, and A. Vaquero Aviles-Casco, Phys. Rev. D 96, 034503 (2017).

[9] A. J. Chambers et al. (QCDSF, UKQCD, and CSSM Collaborations), Phys. Rev. D 96, 114509 (2017).

[10] E. Shintani, K. I. Ishikawa, Y. Kuramashi, S. Sasaki, and T. Yamazaki, Phys. Rev. D 99, 014510 (2019).

[11] F. He and P. Wang, Phys. Rev. D 97, 036007 (2018).

[12] J. M. Alarcón and C. Weiss, Phys. Rev. C 97, 055203 (2018).

[13] T. Gutsche, V. E. Lyubovitskij, and I. Schmidt, Phys. Rev. D 97, 054011 (2018).

[14] C. Mondal, Phys. Rev. D 94, 073001 (2016).

[15] D. Chakrabarti and C. Mondal, Eur. Phys. J. C 73, 2671 (2013).

[16] D. Chakrabarti and C. Mondal, Phys. Rev. D 88, 073006 (2013).

[17] R. S. Sufian, G. F. de Téramond, S. J. Brodsky, A. Deur, and H. G. Dosch, Phys. Rev. D 95, 014011 (2017).

[18] C. Mondal and D. Chakrabarti, Eur. Phys. J. C 75, 261 (2015).

[19] T. Gutsche, V. E. Lyubovitskij, I. Schmidt, and A. Vega, Phys. Rev. D 91, 054028 (2015).

[20] T. Gutsche, V. E. Lyubovitskij, I. Schmidt, and A. Vega, Phys. Rev. D 89, 054033 (2014); 92, 019902(E) (2015).

[21] C. Mondal and D. Chakrabarti, Few Body Syst. 57, 723 (2016).

[22] S. J. Brodsky, G. F. de Teramond, H. G. Dosch, and J. Erlich, Phys. Rep. 584, 1 (2015).
[23] Z. Ye, J. Arrington, R. J. Hill, and G. Lee, Phys. Lett. B 777, 8 (2018).

[24] Z. Abidin and C.E. Carlson, Phys. Rev. D 79, 115003 (2009).

[25] I. C. Cloet and G. A. Miller, Phys. Rev. C 86, 015208 (2012).

[26] B. Pasquini and S. Boffi, Phys. Rev. D 76, 074011 (2007).

[27] C. Alexandrou, S. Bacchio, M. Constantinou, J. Finkenrath, K. Hadjiyiannakou, K. Jansen, G. Koutsou, and A. Vaquero Aviles-Casco, Phys. Rev. D 100, 014509 (2019).

[28] G. D. Cates, C. W. de Jager, S. Riordan, and B. Wojtsekhowski, Phys. Rev. Lett. 106, 252003 (2011).

[29] I. A. Qattan and J. Arrington, Phys. Rev. C 86, 065210 (2012).

[30] M. Diehl and P. Kroll, Eur. Phys. J. C 73, 2397 (2013).

[31] JLab experiment E12-07-108, B. Wojtsekhowski, J. Arrington, S. Gilad, and B. Moffit, spokespersons.

[32] JLab experiment E12-07-109, B. Wojtsekhowski et al., spokespersons.

[33] JLab experiment E12-09-016, B. Wojtsekhowski, G. Cates, and S. Riordan, spokespersons.

[34] JLab experiment E12-09-019, B. Wojtsekhowski, J. Annand, R. Gilman, and B. Quinn, spokespersons.

[35] JLab experiment E12-07-104, W. Brooks, G. Gilfoyle, J. Lachniet, and M. Vineyard, spokespersons.

[36] JLab experiment E12-11-009, J. Arrington, K. Kohl, S. Kowalski, B. Sawatzky, and A. Semenov, spokespersons.

[37] JLab experiment E12-11-106, A. Gasparian, D. Dutta, H. Gao, and M. Khandaker, spokespersons.

[38] R. D. Ball et al. (NNPDF Collaboration), Eur. Phys. J. C 77, 663 (2017).

[39] L. A. Harland-Lang, A. D. Martin, P. Motylinski, and R. S. Thorne, Eur. Phys. J. C 75, 204 (2015).

[40] S. Dulat, T.-J. Hou, J. Gao, M. Guzzi, J. Huston, P. Nadolsky, J. Pumplin, C. Schmidt, D. Stump, and C.-P. Yuan, Phys. Rev. D 93, 033006 (2016).

[41] S. Alekhin, J. Blümlein, S. Moch, and R. Placakyte, Phys. Rev. D 96, 014011 (2017).

[42] A. D. Martin, W. J. Stirling, R. S. Thorne, and G. Watt, Eur. Phys. J. C 63, 189 (2009). 
[43] G. F. de Teramond, T. Liu, R. S. Sufian, H. G. Dosch, S. J. Brodsky, and A. Deur (HLFHS Collaboration), Phys. Rev. Lett. 120, 182001 (2018).

[44] C. A. Aidala, S. D. Bass, D. Hasch, and G. K. Mallot, Rev. Mod. Phys. 85, 655 (2013).

[45] A. Deur, S. J. Brodsky, and G. F. De Téramond, Rep. Prog. Phys. 82, 076201 (2019).

[46] X. Zheng et al. (Jefferson Lab Hall A Collaboration), Phys. Rev. Lett. 92, 012004 (2004).

[47] X. Zheng et al. (Jefferson Lab Hall A Collaboration), Phys. Rev. C 70, 065207 (2004).

[48] D. S. Parno et al. (Jefferson Lab Hall A Collaboration), Phys. Lett. B 744, 309 (2015).

[49] K. V. Dharmawardane et al. (CLAS Collaboration), Phys. Lett. B 641, 11 (2006).

[50] A. Airapetian et al. (HERMES Collaboration), Phys. Rev. Lett. 92, 012005 (2004).

[51] A. Airapetian et al. (HERMES Collaboration), Phys. Rev. D 71, 012003 (2005).

[52] M. G. Alekseev et al. (COMPASS Collaboration), Phys. Lett. B 693, 227 (2010).

[53] D. de Florian, R. Sassot, M. Stratmann, and W. Vogelsang, Phys. Rev. Lett. 101, 072001 (2008).

[54] D. de Florian, R. Sassot, M. Stratmann, and W. Vogelsang, Phys. Rev. D 80, 034030 (2009).

[55] E. R. Nocera, R. D. Ball, S. Forte, G. Ridolfi, and J. Rojo (NNPDF Collaboration), Nucl. Phys. B887, 276 (2014).

[56] P. Jimenez-Delgado, H. Avakian, and W. Melnitchouk (Jefferson Lab Angular Momentum (JAM) Collaboration), Phys. Lett. B 738, 263 (2014).

[57] J. J. Ethier, N. Sato, and W. Melnitchouk, Phys. Rev. Lett. 119, 132001 (2017).

[58] C. D. Roberts, R. J. Holt, and S. M. Schmidt, Phys. Lett. B 727, 249 (2013).

[59] T. Liu, R. S. Sufian, G. F. de Téramond, H. G. Dosch, S. J. Brodsky, and A. Deur, Phys. Rev. Lett. 124, 082003 (2020).

[60] K. F. Liu, Phys. Rev. D 62, 074501 (2000).

[61] K. F. Liu and S. J. Dong, Phys. Rev. Lett. 72, 1790 (1994).

[62] R. Horsley, R. Millo, Y. Nakamura, H. Perlt, D. Pleiter, P. E. L. Rakow, G. Schierholz, A. Schiller, F. Winter, and J. M. Zanotti (QCDSF and UKQCD Collaborations), Phys. Lett. B 714, 312 (2012).

[63] A. J. Chambers, R. Horsley, Y. Nakamura, H. Perlt, P. E. L. Rakow, G. Schierholz, A. Schiller, K. Somfleth, R. D. Young, and J. M. Zanotti, Phys. Rev. Lett. 118, 242001 (2017).

[64] X. Ji, Phys. Rev. Lett. 110, 262002 (2013).

[65] H. W. Lin, J. W. Chen, S. D. Cohen, and X. Ji, Phys. Rev. D 91, 054510 (2015).

[66] C. Alexandrou, K. Cichy, V. Drach, E. Garcia-Ramos, K. Hadjiyiannakou, K. Jansen, F. Steffens, and C. Wiese, Phys. Rev. D 92, 014502 (2015).

[67] C. Alexandrou, K. Cichy, M. Constantinou, K. Hadjiyiannakou, K. Jansen, F. Steffens, and C. Wiese, Phys. Rev. D 96, 014513 (2017).

[68] A. V. Radyushkin, Phys. Rev. D 96, 034025 (2017).

[69] K. Orginos, A. Radyushkin, J. Karpie, and S. Zafeiropoulos, Phys. Rev. D 96, 094503 (2017).
[70] Y. Q. Ma and J. W. Qiu, Phys. Rev. Lett. 120, 022003 (2018).

[71] H. W. Lin et al., Prog. Part. Nucl. Phys. 100, 107 (2018).

[72] R. L. Jaffe and X. D. Ji, Phys. Rev. Lett. 67, 552 (1991).

[73] M. Anselmino, M. Boglione, U. D’Alesio, A. Kotzinian, F. Murgia, A. Prokudin, and S. Melis, Nucl. Phys. B, Proc. Suppl. 191, 98 (2009).

[74] M. Anselmino, M. Boglione, U. D'Alesio, S. Melis, F. Murgia, and A. Prokudin, Phys. Rev. D 87, 094019 (2013).

[75] M. Anselmino, M. Boglione, U. D’Alesio, A. Kotzinian, F. Murgia, A. Prokudin, and C. Turk, Phys. Rev. D 75, 054032 (2007).

[76] Z. B. Kang, A. Prokudin, P. Sun, and F. Yuan, Phys. Rev. D 93, 014009 (2016).

[77] M. Radici, A. Courtoy, A. Bacchetta, and M. Guagnelli, J. High Energy Phys. 05 (2015) 123.

[78] M. Radici, A. M. Ricci, A. Bacchetta, and A. Mukherjee, Phys. Rev. D 94, 034012 (2016).

[79] A. Bacchetta, A. Courtoy, and M. Radici, Phys. Rev. Lett. 107, 012001 (2011).

[80] A. Bacchetta, A. Courtoy, and M. Radici, J. High Energy Phys. 03 (2013) 119.

[81] M. Radici and A. Bacchetta, Phys. Rev. Lett. 120, 192001 (2018).

[82] J. P. Vary, H. Honkanen, J. Li, P. Maris, S. J. Brodsky, A. Harindranath, G. F. de Teramond, P. Sternberg, E. G. Ng, and C. Yang, Phys. Rev. C 81, 035205 (2010).

[83] X. Zhao, H. Honkanen, P. Maris, J. P. Vary, and S. J. Brodsky, Phys. Lett. B 737, 65 (2014).

[84] P. Wiecki, Y. Li, X. Zhao, P. Maris, and J. P. Vary, Phys. Rev. D 91, 105009 (2015).

[85] Y. Li, P. Maris, X. Zhao, and J. P. Vary, Phys. Lett. B 758, 118 (2016).

[86] Y. Li, P. Maris, and J. P. Vary, Phys. Rev. D 96, 016022 (2017).

[87] M. M. Brisudova and S. D. Glazek, Phys. Rev. D 50, 971 (1994).

[88] M. Burkardt, Phys. Rev. D 58, 096015 (1998).

[89] M. Burkardt and A. Langnau, Phys. Rev. D 44, 3857 (1991).

[90] S. J. Brodsky, M. Diehl, and D. S. Hwang, Nucl. Phys. B596, 99 (2001).

[91] O. Gayou et al., Phys. Rev. C 64, 038202 (2001).

[92] O. Gayou et al., Phys. Rev. Lett. 88, 092301 (2002).

[93] J. Arrington, W. Melnitchouk, and J. A. Tjon, Phys. Rev. C 76, 035205 (2007).

[94] T. Pospischil et al., Eur. Phys. J. A 12, 125 (2001).

[95] B. D. Milbrath et al., Phys. Rev. Lett. 80, 452 (1998).

[96] M. K. Jones et al., Phys. Rev. Lett. 84, 1398 (2000).

[97] J. Arrington, Phys. Rev. C 71, 015202 (2005).

[98] N. Bezginov, T. Valdez, M. Horbatsch, A. Marsman, A. C. Vutha, and E. A. Hessels, Science 365, 1007 (2019).

[99] M. Tanabashi et al. (Particle Data Group), Phys. Rev. D 98, 030001 (2018).

[100] R. J. Hill, P. Kammel, W. J. Marciano, and A. Sirlin, Rep. Prog. Phys. 81, 096301 (2018).

[101] D. L. Yao, L. Alvarez-Ruso, and M.J. Vicente-Vacas, Phys. Rev. D 96, 116022 (2017).

[102] C. C. Chang et al., Nature (London) 558, 91 (2018). 
[103] R. Gupta, B. Yoon, T. Bhattacharya, V. Cirigliano, Y. C. Jang, and H. W. Lin, Phys. Rev. D 98, 091501 (2018).

[104] C. Alexandrou et al., Phys. Rev. D 101, 034519 (2020).

[105] M. R. Schindler and S. Scherer, Eur. Phys. J. A 32, 429 (2007).

[106] V. Bernard, L. Elouadrhiri, and U. G. Meissner, J. Phys. G 28, R1 (2002).

[107] H. Hashamipour, M. Goharipour, and S. S. Gousheh, Phys. Rev. D 100, 016001 (2019).

[108] C. Alexandrou, M. Constantinou, S. Dinter, V. Drach, K. Jansen, C. Kallidonis, and G. Koutsou, Phys. Rev. D 88, 014509 (2013).

[109] A. S. Meyer, M. Betancourt, R. Gran, and R. J. Hill, Phys. Rev. D 93, 113015 (2016).

[110] Y. L. Dokshitzer, Zh. Eksp. Teor. Fiz. 73, 1216 (1977) [Sov. Phys. JETP 46, 641 (1977)], http://old.inspirehep .net/record/12615.

[111] V. N. Gribov and L. N. Lipatov, Yad. Fiz. 15, 781 (1972) [Sov. J. Nucl. Phys. 15, 438 (1972)], http://old.inspirehep .net/record/73449.
[112] G. Altarelli and G. Parisi, Nucl. Phys. B126, 298 (1977).

[113] G. P. Salam and J. Rojo, Comput. Phys. Commun. 180, 120 (2009).

[114] J. Lan, C. Mondal, S. Jia, X. Zhao, and J. P. Vary, Phys. Rev. Lett. 122, 172001 (2019).

[115] J. Lan, C. Mondal, S. Jia, X. Zhao, and J. P. Vary, Phys. Rev. D 101, 034024 (2020).

[116] M. Anselmino, M. Boglione, U. D’ Alesio, A. Kotzinian, F. Murgia, A. Prokudin, and S. Melis, Nucl. Phys. B, Proc. Suppl. 191, 98 (2009).

[117] M. Gockeler, Ph. Hägler, R. Horsley, D. Pleiter, P. E. L. Rakow, A. Schäfer, G. Schierholz, and J. M. Zanotti (QCDSF and UKQCD Collaborations), Phys. Lett. B 627, 113 (2005).

[118] I. C. Cloet, W. Bentz, and A. W. Thomas, Phys. Lett. B 659, 214 (2008).

[119] B. Pasquini, M. Pincetti, and S. Boffi, Phys. Rev. D 72, 094029 (2005).

[120] M. Wakamatsu, Phys. Lett. B 653, 398 (2007). 